\title{
A Photometric Survey of Field Stars in the Large Magellanic Cloud: Probing its Star-Formation History ${ }^{1}$
}

\author{
T. A. Smecker-Hane ${ }^{2}$ \\ Dept. of Physics 8 Astronomy, Univ. of California, Irvine, CA 92697, \\ $U S A$
}

J. S. Gallagher, III, Andrew Cole

Astronomy Dept., Univ. of Wisconsin, Madison, WI 53706, USA

P. B. Stetson

National Res. Council of Canada, Herzberg Inst. of Astrophysics, Dominion Astrophysical Obs., Victoria, BC V8X 4M6, Canada

E. Tolstoy

Space Telescope-European Coordinating Facility, Karl-Schwarzschild-Str. 2, D-85748 Garching bei Muenchen, Germany

\section{Introduction}

The Large Magellanic Cloud (LMC) is unique among galaxies in the Local Group in that it is the most massive non-spiral, is relatively gas-rich, and is actively forming stars. Determining its star-formation rate (SFR) as a function of time will be a cornerstone in our understanding of galaxy evolution. The best method of deriving a galaxy's past SFR is to compare the densities of stars in a colormagnitude diagram (CMD), a Hess diagram, with model Hess diagrams. The LMC has a complex stellar population with ages ranging from 0 to $\sim 14 \mathrm{Gyr}$ and metallicities from $-2 \lesssim[\mathrm{Fe} / \mathrm{H}] \lesssim-0.4$, and deriving its SFR and simultaneously constraining model input parameters (distance, age-metallicity relation, reddening, and stellar models) requires well-populated CMDs that span the magnitude range $15 \leq V \leq 24$. Although existing CMDs of field stars in the LMC show tantalizing evidence for a significant burst of star formation that occurred $\sim 3$ Gyr ago (for examples, see Westerlund et al. 1995; Vallenari et al. 1996; Elson, et al. 1997; Gallagher et al. 1999, and references therein), estimates of the enhancement in the SFR vary from factors of 3 to 50 . This uncertainty is caused by the relatively large photometric errors that plague crowded ground-based images, and the small number statistics that plague CMDs created from single Wide Field Planetary Camera 2 (WFPC2) images.

\footnotetext{
${ }^{1}$ Based on observations with the Cerro Tololo Inter-American Observatory and the NASA/ESA Hubble Space Telescope. CTIO is operated by AURA, Inc. under cooperative agreement with the National Science Foundation. The Space Telescope Science Institute is operated by AURA, Inc. under NASA contract 5-26555.
}

${ }^{2}$ Partial funding provided by NSF grant AST-9619460 to TSH. 


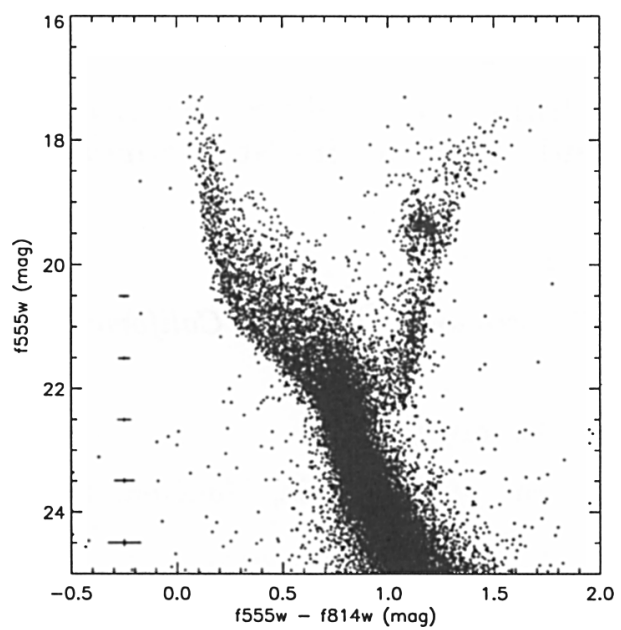

Figure 1. A CMD containing 28,000 stars from one WFPC2 pointing in the LMC bar. (Zero-points have been added to the instrumental magnitudes to approximate V and V-I magnitudes.) Notice the structure in the main-sequence turnoff region at $f 555 \mathrm{w}=21.5$ and $22 \mathrm{mag}$, signs of discontinuities in the SFR and/or age-metallicity relation.

\section{Our Photometric Survey of LMC Areas}

We are combining multicolor imaging of six areas of the LMC obtained with the Cerro-Tololo Inter-American Observatory 1.5-meter telescope with deeper, higher-spatial resolution, WFPC2 imaging in two of these fields. One area lies at the center of the bar (see Figure 1) and one lies in the disk approximately $2^{\circ}$ from the center. Crucial ground-based observations provide data on stars with $\mathrm{V} \lesssim$ 18.5 that are saturated in our WFPC2 images, an accurate calibration of WFPC2 photometry, and help quantify problems such as WFPC2's charge-transfer effect. Our goal is to create CMDs for numerous areas that each will contain $\sim 10^{4}$ stars in the critical magnitude range $20 \leq \mathrm{V} \leq 23$. By modeling Hess diagrams with such a large sample, we can measure a $20 \%$ change in the star-formation rate averaged over 1 Gyr intervals at approximately the 2 -sigma level. By comparing numerous fields in the bar and disk, we can begin to disentangle the histories of the LMC's three distinct components: the bar, the disk, and the thick disk/halo.

\section{References}

Elson, R.A.W., Gilmore, G.F., \& Santiago, B.X. 1997, MNRAS, 289, 157

Gallagher, J.S., III, et al. 1999, this volume

Vallenari, A., et al. 1996, A\&A, 309, 367

Westerlund, B.E., Linde, P., \& Lyngå, G. 1995, A\&A, 298, 39 